\title{
Accretion and evaporation of modified Hayward black hole
}

\author{
Ujjal Debnath $^{\mathrm{a}, \mathrm{b}, \mathrm{c}}$ \\ Department of Mathematics, Indian Institute of Engineering Science and Technology, Shibpur, Howrah 711 103, India
}

Received: 18 February 2015 / Accepted: 3 March 2015 / Published online: 20 March 2015

(C) The Author(s) 2015. This article is published with open access at Springerlink.com

\begin{abstract}
We assume the most general static spherically symmetric black hole metric. The accretion of any general kind of fluid flow around the black hole is investigated. The accretion of the fluid flow around the modified Hayward black hole is analyzed, and we then calculate the critical point, the fluid's four-velocity, and the velocity of sound during the accretion process. Also the nature of the dynamical mass of the black hole during accretion of the fluid flow, taking into consideration Hawking radiation from the black hole, i.e., evaporation of the black hole, is analyzed.
\end{abstract}

\section{Introduction}

At present we live in a Universe which is expanding and the expansion rate is increasing, i.e., the Universe is accelerating, which was confirmed by recent Supernova type Ia observations [1,2]. The large scale structure [3-5] and cosmic microwave background radiation [6] WMAP observations [7-9] also support this acceleration of the Universe. This acceleration is caused by some unknown matter which produces a sufficiently strong negative pressure (with positive energy density), known as dark energy. The present Universe occupies $\sim 4 \%$ ordinary matter, $\sim 74 \%$ dark energy and $\sim 22 \%$ dark matter. Dark energy and dark matter are the two main components in our universe; the present darkenergy and dark-matter densities are $7.01 \times 10^{-27}$ and $2.18 \times 10^{-27} \mathrm{~kg} / \mathrm{m}^{3}$, respectively. The simplest candidate of the dark energy is the cosmological constant $\Lambda$, which obeys the equation of state $\operatorname{EoS} p=w \rho$ with EoS parameter $w=-1[10,11]$. Other candidates for the dark energy are quintessence (where the EoS parameter satisfies $-1<w<$ $-1 / 3)[12,13]$ and phantom (where the EoS parameter sat-

\footnotetext{
a e-mail: ujjaldebnath@yahoo.com

b e-mail: ujjaldebnath@gmail.com

ce-mail: ujjal@iucaa.ernet.in
}

isfies $w<-1)$ [14]. Till now a lot of dark-energy models have been considered. A brief review of dark-energy models is found in Ref. [15].

A condensed object (e.g. a neutron star, a black hole, etc.) surrounded by a fluid can capture particles of the fluid that pass within a certain distance from the condensed object. This phenomenon is termed accretion of the fluid by condensed objects. In Newtonian theory of gravity, the problem of accretion of matter onto the compact object was first formulated by Bondi [16]. Michel [17] first obtained an analytic relativistic accretion (of gas) solution onto the static Schwarzschild black hole. Such accretion processes are candidates to the mechanisms of the formation of supermassive black holes (SMBH) in the center of most active galaxies [18]. In particular, it should show some analogies with the process proposed by Salpeter et al. [19] where galaxies and quasars could get some of their energy from processes of accretion. Using this accretion procedure, Babichev et al. [20,21] formulated the accretion of phantom dark energy onto a static Schwarzschild black hole and showed that static Schwarzschild black hole mass will gradually decrease due to the strong negative pressure of the phantom energy and finally all the masses tend to zero near the big rip singularity. Sun [22] discussed phantom energy accretion onto a black hole in the cyclic universe. Jamil [23] has investigated accretion of a phantom like modified variable Chaplygin gas onto the Schwarzschild black hole. Phantom energy accretion by a stringy charged black hole has been discussed by Sharif et al. [24]. Dark matter and dark energy accretion onto a static black hole has been discussed by Kim et al. [25]. Also the accretion of the dark energy onto the more general Kerr-Newman black hole was studied by Madrid et al. [26]. The new variable modified Chaplygin gas and generalized cosmic Chaplygin gas darkenergy accretions and accretions onto a Kerr-Newman black hole and their features were studied Bhadra et al. [27]. Several authors [28-35] have discussed the accretions of various components of the dark energy onto several types of black holes. 
In the present work, first we assume the most general static spherically symmetric black hole metric in Sect. 2 . The accretion of any general kind of the fluid flow around the black hole will be investigated. The accretion of the fluid flow around the modified Hayward black hole will be analyzed in Sect. 3 and we then calculate the critical point, the fluid's four-velocity, and the velocity of sound during the accretion process. Also the nature of the dynamical mass of the black hole during accretion of the fluid flow and taking into consideration Hawking radiation from the black hole, i.e., evaporation of the black hole, will be analyzed in Sect. 4 . Finally, we shall present fruitful discussions of the accretion of the fluids upon the modified Hayward black hole in Sect. 5 .

\section{Accretion phenomena of general static spherically symmetric black hole}

First we consider general static spherically symmetric metric given by

$\mathrm{d} s^{2}=-A(r) \mathrm{d} t^{2}+\frac{1}{B(r)} \mathrm{d} r^{2}+r^{2}\left(\mathrm{~d} \theta^{2}+\sin \theta \mathrm{d} \phi^{2}\right)$

where $A(r)>0$ and $B(r)>0$ are functions of $r$ only. We can choose $A(r)$ and $B(r)$ in such a way that the above metric represents a black hole metric. Let us assume $M$ is the mass of the black hole. For instance, if $A(r)=B(r)=1-\frac{2 M}{r}$, the above metric represents a Schwarzschild black hole.

The energy-momentum tensor for the fluid is given by

$T_{\mu \nu}=(\rho+p) u_{\mu} u_{\nu}+p g_{\mu \nu}$

where $\rho$ and $p$ are the energy density and pressure of the fluid. The four-velocity vector of the fluid flow is given by $u^{\mu}=\frac{\mathrm{d} x^{\mu}}{\mathrm{d} s}=\left(u^{0}, u^{1}, 0,0\right)$ where $u^{0}$ and $u^{1}$ are the nonzero components of velocity vector satisfying $u_{\mu} u^{\mu}=-1$. This implies $g_{00} u^{0} u^{0}+g_{11} u^{1} u^{1}=-1$. So we can obtain $\left(u^{0}\right)^{2}=\frac{\left(u^{1}\right)^{2}+B}{A B}$ and let the radial velocity of the flow $u^{1}=$ $u$, thus we have $u_{0}=g_{00} u^{0}=\sqrt{\frac{A}{B}} \sqrt{u^{2}+B}$. Here $\sqrt{-g}=$ $\sqrt{\frac{A}{B}} r^{2} \sin \theta$. From the above Eq. (2), we obtain $T_{0}^{1}=(\rho+$ p) $u_{0} u$. It is assumed that $u<0$ for inward flow of the fluid toward the black hole.

In the fluid flow, we may assume that the fluid is dark matter or any kind of dark energy. A proper dark-energy accretion model for a static spherically symmetric black hole should be obtained by generalizing Michel's theory [17]. In the dark-energy accretion onto Schwarzschild black hole, Babichev et al. [20,21] have performed the above generalization. We shall follow now the above procedure in the case of static spherically symmetric black hole. The relativistic Bernoulli equation (the time component) of the energy-momentum conservation law is $T_{; v}^{\mu \nu}=0$, and we obtain $\frac{\mathrm{d}}{\mathrm{d} r}\left(T_{0}{ }^{1} \sqrt{-g}\right)=0$, which provides the first integral, $(\rho+p) u_{0} u^{1} \sqrt{-g}=C_{1}$. This simplifies to

$u r^{2} M^{-2}(\rho+p) \frac{A}{B} \sqrt{u^{2}+B}=C_{1}$

where $C_{1}$ is an integration constant, which has the dimension of the energy density. Moreover, the energy flux equation can be derived by the projection of the conservation law for the energy-momentum tensor onto the fluid four-velocity, i.e., $u_{\mu} T_{; v}^{\mu \nu}=0$, which gives $u^{\mu} \rho_{, \mu}+(\rho+p) u_{; \mu}^{\mu}=0$. From this, we obtain

$u r^{2} M^{-2} \sqrt{\frac{A}{B}} \exp \left[\int_{\rho_{\infty}}^{\rho_{\mathrm{h}}} \frac{\mathrm{d} \rho}{\rho+p(\rho)}\right]=-C$

where $C$ is an integration constant (energy flux onto the black hole) and the associated minus sign is taken for convenience. Also $\rho_{\mathrm{h}}$ and $\rho_{\infty}$ represent the energy densities at the black hole horizon and at infinity, respectively. Combining Eqs. (3) and (4), we obtain

$(\rho+p) \sqrt{u^{2}+B} \sqrt{\frac{A}{B}} \exp \left[-\int_{\rho_{\infty}}^{\rho_{\mathrm{h}}} \frac{\mathrm{d} \rho}{\rho+p(\rho)}\right]=C_{2}$

where $C_{2}=-C_{1} / C=\rho_{\infty}+p\left(\rho_{\infty}\right)$. The equation of mass flux $J_{; \mu}^{\mu}=0$ is given by $\frac{\mathrm{d}}{\mathrm{d} r}\left(J^{1} \sqrt{-g}\right)=0$, which integrates to $\rho u^{1} \sqrt{-g}=A_{1}$ and yields

$\rho u r^{2} M^{-2} \sqrt{\frac{A}{B}}=C_{3}$

where $C_{3}$ is an integration constant. From (3) and (6), we obtain

$\frac{\rho+p}{\rho} \sqrt{\frac{A}{B}} \sqrt{u^{2}+B}=\frac{C_{1}}{C_{3}}=C_{4}=$ constant.

Now let us assume

$V^{2}=\frac{\mathrm{d} \ln (\rho+p)}{\mathrm{d} \ln \rho}-1$.

Thus, from Eqs. (6), (7), and (8), we obtain

$$
\begin{aligned}
& {\left[V^{2}-\frac{u^{2}}{u^{2}+B}\right] \frac{\mathrm{d} u}{u}+\left[-2 V^{2}+\frac{1}{2}\left(\frac{A^{\prime}}{A}-\frac{B^{\prime}}{B}\right)\right.} \\
& \left.\times\left(V^{2}+1\right) r+\frac{r B^{\prime}}{2\left(u^{2}+B\right)}\right] \frac{\mathrm{d} r}{r}=0 .
\end{aligned}
$$

Now if one or the other of the bracketed terms in (9) vanishes, we get a turn-around point, and in this case, the solutions will be the double-valued in either $r$ or $u$. There are only solutions which pass through a critical point that correspond to material falling into (or flowing out of) the object with monotonically increasing velocity along with the particle trajectory. A point where the speed of the flow is equal to the speed of sound is called a critical point. It is assumed that the critical point of accretion is located at $r=r_{\mathrm{c}}$, which is obtained by taking 
the two bracketed terms (coefficients of $\mathrm{d} u$ and $\mathrm{d} r$ ) in Eq. (9) to be zero. So at the critical point, we obtain

$V_{\mathrm{c}}^{2}=\frac{u_{\mathrm{c}}^{2}}{u_{\mathrm{c}}^{2}+B\left(r_{\mathrm{c}}\right)}$

and

$\frac{4 V_{\mathrm{c}}^{2}}{r_{\mathrm{c}}}=\left[\frac{A^{\prime}\left(r_{\mathrm{c}}\right)}{A\left(r_{\mathrm{c}}\right)}-\frac{B^{\prime}\left(r_{\mathrm{c}}\right)}{B\left(r_{\mathrm{c}}\right)}\right]\left(V_{\mathrm{c}}^{2}+1\right)+\frac{B^{\prime}\left(r_{\mathrm{c}}\right)}{u_{\mathrm{c}}^{2}+B\left(r_{\mathrm{c}}\right)}$.

Here, the subscript $c$ denotes the critical value and $u_{\mathrm{c}}$ is the critical speed of the flow at the critical point $r_{\mathrm{c}}$. From the above two expressions, we have

$u_{\mathrm{c}}^{2}=\frac{B^{\prime}\left(r_{\mathrm{c}}\right)}{2} \frac{A^{\prime}\left(r_{\mathrm{c}}\right)}{A\left(r_{\mathrm{c}}\right)}\left[\frac{2}{r_{\mathrm{c}}}-\frac{A^{\prime}\left(r_{\mathrm{c}}\right)}{A\left(r_{\mathrm{c}}\right)}+\frac{B^{\prime}\left(r_{\mathrm{c}}\right)}{B\left(r_{\mathrm{c}}\right)}\right]^{-1}$

and

$V_{\mathrm{c}}^{2}=\left[1+2 \frac{A\left(r_{\mathrm{c}}\right)}{A^{\prime}\left(r_{\mathrm{c}}\right)} \frac{B(r)}{B^{\prime}\left(r_{\mathrm{c}}\right)}\left(\frac{2}{r_{\mathrm{c}}}-\frac{A^{\prime}\left(r_{\mathrm{c}}\right)}{A\left(r_{\mathrm{c}}\right)}+\frac{B^{\prime}\left(r_{\mathrm{c}}\right)}{B\left(r_{\mathrm{c}}\right)}\right)\right]^{-1}$.

At the critical point $r_{\mathrm{c}}$, the sound speed can be determined by

$c_{\mathrm{s}}^{2}=\left.\frac{\mathrm{d} p}{\mathrm{~d} \rho}\right|_{r=r_{\mathrm{c}}}=\frac{C_{4} V_{\mathrm{c}}\left(V_{\mathrm{c}}^{2}+1\right)}{u_{\mathrm{c}}} \sqrt{\frac{B\left(r_{\mathrm{c}}\right)}{A\left(r_{\mathrm{c}}\right)}}-1$.

The physically acceptable solutions of the above equations may be obtained if $u_{\mathrm{c}}^{2}>0$ and $V_{\mathrm{c}}^{2}>0$, which leads to

$A^{\prime}\left(r_{\mathrm{c}}\right) B^{\prime}\left(r_{\mathrm{c}}\right)>0$ and $\frac{2}{r_{\mathrm{c}}}>\frac{A^{\prime}\left(r_{\mathrm{c}}\right)}{A\left(r_{\mathrm{c}}\right)}-\frac{B^{\prime}\left(r_{\mathrm{c}}\right)}{B\left(r_{\mathrm{c}}\right)}$.

From the above equation we can obtain the bound of $r_{\mathrm{c}}$ if $A$ and $B$ are known for several kinds of static black holes.

\section{Accretion phenomena of modified Hayward black hole}

The static spherically symmetric space-time is described by the Hayward metric which is obtained by $A(r)=B(r)$ in Eq. (1) and is given by [36]

$\mathrm{d} s^{2}=-B(r) \mathrm{d} t^{2}+\frac{1}{B(r)} \mathrm{d} r^{2}+r^{2}\left(\mathrm{~d} \theta^{2}+\sin \theta \mathrm{d} \phi^{2}\right)$.

Here, $M$ is the mass of the Hayward black hole and $B(r)=$ $1-\frac{2 M r^{2}}{r^{3}+2 M l^{2}}$, where $l$ is a parameter with dimensions of length (Hubble length) with a small scale related to the inverse cosmological constant $\Lambda$ ( $l$ is a convenient encoding of the central energy density $\frac{3}{8 \pi l^{2}} \sim \Lambda$, assumed positive). Such a behavior has been proposed by Sakharov $[37,38]$ as the equation of state of matter at high density and by Markov $[39,40]$ based on an upper limit on the density or curvature, to be ultimately justified by a quantum theory of gravity. In the limit $r \rightarrow \infty, B(r) \approx 1-\frac{2 M}{r}$, which represents the
Schwarzschild black hole, but it becomes a de Sitter black hole as $B(r) \approx 1-\frac{r^{2}}{l^{2}}$ near the center $(r \approx 0)$, so it is a regular space-time without singularity. Thus a Hayward black hole is the simplest regular black hole. Some physical consequences of Hayward black holes have been discussed by several authors [41-43]. After that the Hayward metric was modified [44] by choosing $A(r)=f(r) B(r)$, satisfying the following conditions: it

(i) preserves the Schwarzschild behavior at large $r$,

(ii) includes the one-loop quantum corrections, and

(iii) allows for a finite time dilation between the center and infinity.

So the modified Hayward black hole metric is given by [44]

$\mathrm{d} s^{2}=-f(r) B(r) \mathrm{d} t^{2}+\frac{1}{B(r)} \mathrm{d} r^{2}+r^{2}\left(\mathrm{~d} \theta^{2}+\sin \theta \mathrm{d} \phi^{2}\right)$

where

$B(r)=1-\frac{2 M r^{2}}{r^{3}+2 M l^{2}}, \quad f(r)=1-\frac{\alpha \beta M}{\alpha r^{3}+\beta M}$

with $\alpha, \beta$ positive constants. Now from the relation $A(r)=$ $f(r) B(r)$, we may obtain

$\frac{A^{\prime}(r)}{A(r)}=\frac{f^{\prime}(r)}{f(r)}+\frac{B^{\prime}(r)}{B(r)}$.

Also from the expressions of $B(r)$ and $f(r)$ [Eq. (18)], we get

$\frac{B^{\prime}(r)}{B(r)}=\frac{2 M r\left(r^{3}-4 M l^{2}\right)}{\left(r^{3}+2 M l^{2}\right)\left[r^{3}+2 M\left(l^{2}-r^{2}\right)\right]}$,

$\frac{f^{\prime}(r)}{f(r)}=\frac{3 \alpha^{2} \beta M r^{2}}{\left(\alpha r^{3}+\beta M\right)\left[\alpha r^{3}+(1-\alpha) \beta M\right]}$.

Since outside the horizon,

(i) $B(r)>0$, which implies $r^{3}>2 M\left(r^{2}-l^{2}\right)$ and (ii) $f(r)>0$,

we get $r>\left[\frac{\beta(\alpha-1) M}{\alpha}\right]^{\frac{1}{3}}$ with $\alpha>1$. So from Eq. (21), we have $f^{\prime}(r)>0$.

If we assume that the fluid flow accretes upon the modified Hayward black hole, we can calculate the expressions of $u_{\mathrm{c}}^{2}, V_{\mathrm{c}}^{2}$ and $c_{s}^{2}$ at the critical point $r_{\mathrm{c}}$. The expressions are given below (using Eqs. (12), (13), and (14)):

$$
\begin{aligned}
u_{\mathrm{c}}^{2}= & \frac{B^{\prime}\left(r_{\mathrm{c}}\right)}{2}\left(\frac{B^{\prime}\left(r_{\mathrm{c}}\right)}{B\left(r_{\mathrm{c}}\right)}+\frac{f^{\prime}\left(r_{\mathrm{c}}\right)}{f\left(r_{\mathrm{c}}\right)}\right)\left(\frac{2}{r_{\mathrm{c}}}-\frac{f^{\prime}\left(r_{\mathrm{c}}\right)}{f\left(r_{\mathrm{c}}\right)}\right)^{-1}, \\
V_{\mathrm{c}}^{2}= & {\left[1+2 \frac{B\left(r_{\mathrm{c}}\right)}{B^{\prime}\left(r_{\mathrm{c}}\right)}\left(\frac{B^{\prime}\left(r_{\mathrm{c}}\right)}{B\left(r_{\mathrm{c}}\right)}+\frac{f^{\prime}\left(r_{\mathrm{c}}\right)}{f\left(r_{\mathrm{c}}\right)}\right)^{-1}\right.} \\
& \left.\times\left(\frac{2}{r_{\mathrm{c}}}-\frac{f^{\prime}\left(r_{\mathrm{c}}\right)}{f\left(r_{\mathrm{c}}\right)}\right)\right]^{-1}, \\
c_{\mathrm{s}}^{2}= & \frac{C_{4} V_{\mathrm{c}}\left(V_{\mathrm{c}}^{2}+1\right)}{u_{\mathrm{c}} \sqrt{f\left(r_{\mathrm{c}}\right)}}-1
\end{aligned}
$$


where $B(r), f(r)$, and their derivatives are given in (18), (20), and (21) at the point $r=r_{\mathrm{c}}$. The physically acceptable solutions of the above equations may be obtained if $u_{\mathrm{c}}^{2}>0$ and $V_{\mathrm{c}}^{2}>0$, which leads to

$B^{\prime}\left(r_{\mathrm{c}}\right)>0$ and $0<\frac{f^{\prime}\left(r_{\mathrm{c}}\right)}{f\left(r_{\mathrm{c}}\right)}<\frac{2}{r_{\mathrm{c}}}$.

From the above restrictions, we may get the bounds of $r_{\mathrm{c}}$, that is $(\alpha>1)$ :

$r_{\mathrm{c}}^{3}>\operatorname{Max}\left\{4 M l^{2}, \frac{\beta(-4+5 \alpha+\sqrt{\alpha(25 \alpha-24)}) M}{4 \alpha}\right\}$.

For example, we assume the fluid flow obeys a linear equation of state $p=w \rho(w=$ constant $)$ as it accretes upon the modified Hayward black hole. Then we obtain $c_{\mathrm{s}}^{2}=w$ and $V_{\mathrm{c}}^{2}=0$ and from (10), we obtain $u_{\mathrm{c}}=0$. From Eqs. (22) and (24), we see that the critical point occurs at the point $r_{\mathrm{c}}=$ $\left(4 M l^{2}\right)^{\frac{1}{3}}$. For the general equation of state where $w=w(t)$, we obtain $c_{\mathrm{s}}^{2} \neq$ constant, $V_{\mathrm{c}}^{2} \neq 0$, and $u_{\mathrm{c}}^{2} \neq 0$. In this case, it is very difficult to obtain the critical point $r_{\mathrm{c}}$.

\section{Changes of black hole mass during accretion and evaporation}

The rate of change of mass $\dot{M}$ of the black hole is computed by integrating the flux of the fluid over the 2-dimensional surface of the black hole and given by $\dot{M}=-\int T_{0}^{1} \mathrm{~d} S$ where $\mathrm{d} S=\sqrt{-g} \mathrm{~d} \theta \mathrm{d} \phi$. Using Eq. (3), we obtain the rate of change of the mass of the black hole in the following form:

$\dot{M}=4 \pi C M^{2}\left(\rho_{\infty}+p\left(\rho_{\infty}\right)\right)$.

The above result is also valid for any equation of state $p=$ $p(\rho)$. So the rate of change of mass for the accreting fluid around the black hole will be

$\dot{M}_{\mathrm{acc}}=4 \pi C M^{2}(\rho+p)$.

We see that the rate of change of mass for the general spherically symmetric static black hole due to accretion of the fluid flow becomes exactly the rate of the case of a Schwarzschild black hole. From the expression (28) it is to be noted that the rate of change of the mass of any static spherically symmetric black hole is completely independent of $A(r)$ and $B(r)$. When some fluid accretes outside the black hole, the mass function $M$ of the black hole is considered as a dynamical mass function and hence it should be a function of time also. So $\dot{M}$ is time dependent and the increasing or decreasing of the black hole mass $M$ sensitively depends on the nature of the fluid which accretes upon the black hole. If $\rho+p<0$ i.e., for phantom dark-energy accretion, the mass of the black hole decreases but if $\rho+p>0$, i.e., for quintessence darkenergy accretion, the mass of the black hole increases.
We may also assume that the black hole evaporates by the Hawking radiation process. The rate of change of mass for the evaporation is given by

$\dot{M}_{\mathrm{eva}}=-\frac{D}{M^{2}}$

where $D>0$ is a constant whose value depends on the model [45]. Now due to accretion of the fluid flow and evaporation of the mass of the black hole, we get the rate of change of the mass of the black hole as

$\dot{M}=\dot{M}_{\mathrm{acc}}+\dot{M}_{\mathrm{eva}}=4 \pi C M^{2}(\rho+p)-\frac{D}{M^{2}}$.

For the accretion scenario, the change of the mass of the black hole completely depends on the nature of the fluid that accretes. But for an evaporation process, the change of the mass of the black hole is independent of the nature of the fluid, because this is internal process. In fact, when the accretion fluid is only the cosmological constant $(p=-\rho)$, the mass of the black hole for only the accretion scenario is always the same throughout the time evolution. Only in the accretion process, the mass of the black hole increases for a normal fluid and quintessence type dark energy fluid and decreases for phantom dark energy. But due to accretion as well as evaporation, $\dot{M}>0$ for $M^{4}>\frac{D}{4 \pi C(\rho+p)}$ and $\dot{M}<0$ for $M^{4}<\frac{D}{4 \pi C(\rho+p)}$ for a normal fluid and a quintessence type dark energy, but for phantom energy, the black hole mass always decreases $(\dot{M}<0)$. Thus evaporation supports the decreasing of the mass of the black hole with some restrictions of the minimum values of the mass of the black hole.

\section{Discussions and concluding remarks}

First we have assumed the most general static spherically symmetric black hole metric. The accretion of any general kind of the fluid flow around the black hole has been investigated. For this general kind of static black hole, the critical point, velocity of sound, and the fluid's four-velocity have been calculated and it was shown that these values depend completely on the metric coefficients. Next, the accretion of the fluid flow around the modified Hayward black hole has been analyzed and we then calculated the critical point, the fluid's four-velocity, and the velocity of sound during the accretion process. We can mention that outside the horizon, (i) $B(r)>0$, which implies $r^{3}>2 M\left(r^{2}-l^{2}\right)$, and (ii) $f(r)>0$, so we get $r>\left[\frac{\beta(\alpha-1) M}{\alpha}\right]^{\frac{1}{3}}$ with $\alpha>1$ and also $f^{\prime}(r)>0$. For the physical region of accretion the bounds of the critical point have been generated and were found to be $r_{\mathrm{c}}^{3}>\operatorname{Max}\left\{4 M l^{2}, \frac{\beta(-4+5 \alpha+\sqrt{\alpha(25 \alpha-24)}) M}{4 \alpha}\right\}$. When the perfect fluid satisfies a linear equation of state, $p=w \rho(w=$ constant), it accretes upon the modified Hayward black hole, 
we have obtained: $c_{s}^{2}=w, u_{\mathrm{c}}=0$, and $V_{\mathrm{c}}^{2}=0$. In this accretion process, we have seen that the critical point occurs at the point $r_{\mathrm{c}}=\left(4 M l^{2}\right)^{\frac{1}{3}}$. Also the nature of the dynamical mass of the black hole during accretion of the fluid flow, taking into consideration Hawking radiation from the black hole, i.e., evaporation of the black hole, has been analyzed. Only in the accretion process, the mass of the black hole increases for a normal fluid and a quintessence type dark-energy fluid and decreases for phantom dark energy. But due to accretion as well as evaporation, $\dot{M}>0$ for $M^{4}>\frac{D}{4 \pi C(\rho+p)}$ and $\dot{M}<0$ for $M^{4}<\frac{D}{4 \pi C(\rho+p)}$ for a normal fluid and a quintessence type dark energy, but for phantom energy, the black hole mass always decreases $(\dot{M}<0)$. Thus evaporation supports the decreasing of the mass of the black hole with some restrictions on the minimum values of the mass of the black hole.

Acknowledgments The author is thankful for warm hospitality to IUCAA, Pune, India, where the work was carried out.

Open Access This article is distributed under the terms of the Creative Commons Attribution License which permits any use, distribution, and reproduction in any medium, provided the original author(s) and the source are credited.

Funded by $\mathrm{SCOAP}^{3}$ / License Version CC BY 4.0.

\section{References}

1. A.G. Riess et al., Supernova Search Team Collaboration, Astron. J. 116, 1009 (1998)

2. S. Perlmutter et al., Supernova Cosmology Project Collaboration, Astrophys. J. 517, 565 (1999)

3. M. Tegmark et al., SDSS Collaboration, Phys. Rev. D 69, 103501 (2004)

4. K. Abazajian et al., SDSS Collaboration, Astron. J. 128, 502 (2004)

5. K. Abazajian et al., Astron. J. 129, 1755 (2005)

6. D.N. Spergel et al., Astrophys. J. Suppl. 170, 377 (2007)

7. D.N. Spergel et al., WMAP Collaboration, Astrophys. J. Suppl. Ser. 148, 175 (2003)

8. S. Briddle et al., Science 299, 1532 (2003)

9. C.L. Bennett et al., Astrophys. J. Suppl. 148, 1 (2003)
10. T. Padmanabhan, Phys. Rep. 380, 235 (2003)

11. V. Sahni, A.A. Starobinsky, Int. J. Mod. Phys. D 9, 373 (2000)

12. P.J.E. Peebles, B. Ratra, Astrophys. J. 325, L17 (1988)

13. R.R. Caldwell, R. Dave, P.J. Steinhardt, Phys. Rev. Lett. 80, 1582 (1998)

14. R.R. Caldwell, Phys. Lett. B 545, 23 (2002)

15. E.J. Copeland, M. Sami, S. Tsujikawa, Int. J. Mod. Phys. D 15, 1753 (2006)

16. H. Bondi, Mon. Not. R. Astron. Soc. 112, 195 (1952)

17. F.C. Michel, Astrophys. Space Sci. 15, 153 (1972)

18. J.W. Moffat. arXiv:astro-ph/9704232

19. D. Merritt, L. Ferrarese. arXiv:astro-ph/0107134v2

20. E. Babichev et al., Phys. Rev. Lett. 93, 021102 (2004)

21. E. Babichev, V. Dokuchaev, Y. Eroshenko, J. Exp. Theor. Phys. 100, 528 (2005)

22. C.Y. Sun, Phys. Rev. D 78, 064060 (2008)

23. M. Jamil, Eur. Phys. J. C 62, 609 (2009)

24. M. Sharif, G. Abbas, Chin. Phys. Lett. 29, 010401 (2012)

25. S.W. Kim, Y. Kang, Int. J. Mod. Phys. Conf. Ser. 12, 320 (2012)

26. J.A. Jiḿenez Madrid, P.F. Gonźalez-Díaz, Grav. Cosmol. 14, 213 (2008)

27. J. Bhadra, U. Debnath, Eur. Phys. J. C 72, 1912 (2012)

28. B. Nayak, M. Jamil, Phys. Lett. B 709, 118 (2012)

29. D. Dwivedee, B. Nayak, M. Jamil, L.P. Singh. arXiv:1110.6350v1 [gr-qc]

30. J.A.S. Lima, D.C. Guariento, J.E. Horvath, Phys. Lett. B 693, 218 (2010)

31. M. Sharif, G. Abbas, Chin. Phys. Lett. 28, 090402 (2011)

32. P. Martín-Moruno et al. arXiv:0803.2005v1 [gr-qc]

33. M.G. Rodrigues, A.E. Bernardiniz. arXiv:1208.1572v1 [gr-qc]

34. G. Abhas. arXiv:1309.0807v1 [gr-qc]

35. P. Martín-Moruno et al. arXiv:astro-ph/0603761

36. S.A. Hayward, Phys. Rev. Lett. 96, 031103 (2006)

37. A.D. Sakharov, Sov. Phys. JETP 22, 241 (1966)

38. E.B. Gliner, Sov. Phys. JETP 22, 378 (1966)

39. M.A. Markov, JETP Lett. 36, 265 (1982)

40. V.P. Frolov, M.A. Markov, V.F. Mukhanov, Phys. Rev. D 41, 383 (1990)

41. G. Abbas, U. Sabiullah, Astrophys. Space Sci. 352, 769 (2014)

42. K. Lin, J. Li, S. Yang, Int. J. Theor. Phys. 52, 3771 (2013)

43. M. Halilsoya, A. Ovgunb, S.H. Mazharimousavic, Eur. Phys. J. C 74, 2796 (2014)

44. T. De Lorenzo, C. Pacilioy, C. Rovelli, S. Speziale. arXiv:1412.6015 [gr-qc]

45. D.B. Cline, D.A. Sanders, W. Hong, Astrophys. J. 486, 169 (1997) 